\title{
A novel method for simple detection of mutations conferring drug resistance in Mycobacterium leprae, based on a DNA microarray, and its applicability in developing countries
}

\begin{abstract}
Correspondence Masanori Matsuoka matsuoka@nih.go.jp
\end{abstract}

Received 14 April 2008 Accepted 18 June 2008

\author{
Masanori Matsuoka, ${ }^{1} \dagger$ Khin Saw Aye, ${ }^{2}$ Kyaw Kyaw, ${ }^{3}$ \\ Esterlina Virtudes Tan, ${ }^{4}$ Ma Victoria Balagon, ${ }^{4}$ Paul Saunderson, ${ }^{4}$ \\ Robert Gelber, ${ }^{4}$ Masanao Makino, ${ }^{5}$ Chie Nakajima ${ }^{6}$ and Yasuhiko Suzuki ${ }^{6} \dagger$ \\ ${ }^{1}$ Leprosy Research Center, National Institute of Infectious Diseases, Tokyo, Japan \\ ${ }^{2}$ Department of Medical Research, Yangon, Myanmar \\ ${ }^{3}$ Central Special Skin Clinic, Yangon General Hospital, Yangon, Myanmar \\ ${ }^{4}$ Leonard Wood Memorial, Cebu, The Philippines \\ ${ }^{5}$ National Leprosy Hospital Okukomyoen, Okayama, Japan \\ ${ }^{6}$ Department of Global Epidemiology, Research Center for Zoonosis Control, Hokkaido University, \\ Sapporo, Japan
}

\begin{abstract}
A simple method to detect mutations in the genome of Mycobacterium leprae that confer resistance to key drugs for leprosy was exploited on the basis of a reverse hybridization system. A series of oligonucleotide probes corresponding to each mutation in the folP1, rpo $B$ and gyrA genes for dapsone, rifampicin and ofloxacin resistance, respectively, were selected and fixed on a glass slide as capture probes, to develop a DNA microarray termed the leprosy drug susceptibility-DNA microarray (LDS-DA). Mutations in clinical isolates of $M$. leprae were successfully identified by the LDS-DA. Feasibility studies were conducted to evaluate the performance of the LDS-DA in two developing countries, Myanmar and the Philippines. The high concordance of the results obtained by this method with the results of nucleotide sequencing strongly supports the applicability of the LDS-DA as a drug susceptibility test in place of sequencing, a time-consuming and costly procedure. This is a rapid and simple method for the simultaneous susceptibility testing of three front-line drugs for leprosy, and solves the problems of previously reported methods.
\end{abstract}

\section{INTRODUCTION}

The current strategy for leprosy control relies mainly on multidrug therapy (MDT) (WHO, 1998). However, cases of leprosy caused by drug-resistant Mycobacterium leprae have been documented as the result of therapeutic failure (Cambau et al., 2001; Maeda et al., 2001; Matsuoka et al., 2000, 2003). Although information on the drug susceptibility of clinical isolates contributes to the better outcome of treatment, susceptibility testing has rarely been done because of its difficulty. Antibiotic susceptibility testing of $M$. leprae still relies on a time-consuming method based on the growth of bacteria in mouse footpads (Shepard, 1960),

†These authors contributed equally to this work.

Abbreviations: $\mathrm{Bl}$, bacterial index; DRDR, drug-resistance-determining region; LDS-DA, leprosy drug susceptibility-DNA microarray; MDT, multidrug therapy. which takes up to 12 months to give a result. This has hindered the comprehensive surveillance that would offer useful information to evaluate the efficacy of MDT and to prevent the spread of drug-resistant strains. Recent advances in the molecular biology of drug-resistant $M$. leprae have enabled the development of drug susceptibility tests for key component drugs of MDT, by the detection of relevant gene mutations that confer resistance (Williams \& Gillis, 2004). The molecular mechanism of rifampicin resistance was first demonstrated in Escherichia coli and thereafter in M. leprae (Honoré \& Cole, 1993). Rifampicin resistance is strongly correlated with mutations in the $r p o B$ gene, encoding the $\beta$ subunit of RNA polymerase (Honoré \& Cole, 1993; Honoré et al., 1993; Williams et al., 1994, 2001; Matsuoka et al., 2000, 2003; Cambau et al., 2001; Maeda et al., 2001; Zhang et al., 2004). Resistance to fluoroquinolones has been proved to correlate with 
mutations in the gyrA gene, encoding the A subunit of DNA gyrase in M. leprae (Cambau et al., 1997; Matsuoka et al., 2000; Cambau et al., 2001), as in many other bacteria. In addition, mutations in the folP1 gene, encoding dihydrofolate synthetase, have been shown to be responsible for dapsone resistance (Kai et al., 1999; Matsuoka et al., 2000, 2003, 2007; Williams et al., 2000; Lee et al., 2001; Maeda et al., 2001; Cambau et al., 2006). The prevalence of drug resistance in selected areas was surveyed through the application of molecular analysis to detect mutations conferring drug resistance (Matsuoka et al., 2007). Analysis of mutations is generally performed by sequencing the target genomic region, amplified by PCR, although the implementation of sequencing is not easy in many developing countries. Therefore, a simple and rapid method to detect mutations conferring drug resistance has been long awaited. In the current study, a DNA microarray method was developed and the applicability of this system was evaluated in Myanmar and the Philippines.

\section{METHODS}

Design of capture probes. Mutant nucleotide sequences conferring resistance to dapsone, rifampicin and ofloxacin and their corresponding wild-type sequences in Mycobacterium leprae (Table 1) were employed in this study. Nearly all the drug-resistant strains of $M$. leprae reported so far are covered by the mutations selected. Capture oligonucleotide probes (14- to 18-mer) for the detection of the mutations were designed according to these data. Optimal sequences of oligonucleotides corresponding to each missense mutation were designed empirically as shown in Fig. 1(a). The array of capture oligonucleotide probes was covalently bound to the surface of a glass slide coated with polycarbodiimide and the resulting DNA microarray was designated the leprosy drug susceptibility-DNA microarray (LDSDA), as shown in Fig. 1(b).

Amplification of three target gene fragments. Target regions of folP1 (accession no. AL 583917, gene ML583917), rpoB (accession no. AL583923, gene ML1891) and gyrA (accession no. AL583917, gene ML0006) were simultaneously amplified with three primer pairs in one PCR. The sequences of the primers are listed in Table 2. PCR was carried out using the G mixture of the FailSafe PCR System (EPICENTRE) in a volume of $25 \mu \mathrm{l}$ with $1 \mu \mathrm{M}$ of each primer. Cycling conditions began with an initial incubation at $94{ }^{\circ} \mathrm{C}$ for $4 \mathrm{~min}$, followed by 40 cycles of annealing at $58{ }^{\circ} \mathrm{C}$ for $30 \mathrm{~s}$, extension at $72{ }^{\circ} \mathrm{C}$ for $30 \mathrm{~s}$, and denaturation at $94{ }^{\circ} \mathrm{C}$ for $30 \mathrm{~s}$. Finally, incomplete PCR products were extended for $5 \mathrm{~min}$ at $72{ }^{\circ} \mathrm{C}$. The amplified DNA fragments were confirmed by gel electrophoresis through $4.0 \%$ Metaphor Agarose (FMC Corp.) in TBE (Tris/borate/ EDTA, pH 8.0) buffer.

LDS-DA assay. A $2 \mu \mathrm{l}$ aliquot of the resulting PCR mixture was mixed with $38 \mu \mathrm{l}$ UniHyb Hybridization Solution (TeleChem International), heat denatured at $98{ }^{\circ} \mathrm{C}$ for 5 min and quickly chilled. The solution was then applied to the LDS-DA and incubated at $42{ }^{\circ} \mathrm{C}$ for $60 \mathrm{~min}$ followed by stringent washing with $50 \mu \mathrm{l}$ washing solution (3 M tetramethylammonium chloride; Sigma-Aldrich) at $47{ }^{\circ} \mathrm{C}$ for $60 \mathrm{~min}$. The biotin-labelled DNA fragments hybridizing to the capture probes on the LDS-DA were detected by avidin-biotinhorseradish peroxidase complex (VECTASTAIN Elite ABC kit, Vector

Table 1. Missense mutations associated with drug resistance in $M$. leprae

\begin{tabular}{|c|c|c|c|c|c|c|c|}
\hline \multirow[t]{2}{*}{ Drug } & \multirow[t]{2}{*}{ Gene } & \multirow[t]{2}{*}{ Codon no. } & \multicolumn{2}{|c|}{ Susceptible } & \multicolumn{2}{|c|}{ Resistant } & \multirow[t]{2}{*}{ References $\dagger$} \\
\hline & & & Codon & $\mathrm{AA}^{*}$ & Codon & $\mathrm{AA}^{*}$ & \\
\hline \multirow[t]{8}{*}{ Dapsone } & folp1 & 53 & ACC & Thr & GCC & Ala & $1,2,3$ \\
\hline & & & & & GTC & Val & 4 \\
\hline & & & & & ATC & Ile & $2,3,5,6$ \\
\hline & & & & & AGG & Arg & 3 \\
\hline & & & & & AGA & Arg & 4 \\
\hline & & 55 & CCC & Pro & TCC & Ser & 1,7 \\
\hline & & & & & CGC & Arg & 1,3 \\
\hline & & & & & СTC & Leu & $2,3,8$ \\
\hline \multirow[t]{9}{*}{ Rifampicin } & $r p o B$ & 407 & CAG & Gln & GTG & Val & 9 \\
\hline & & 410 & GAT & Asp & AAT & Asn & 2 \\
\hline & & & & & TAT & Tyr & 8 \\
\hline & & 420 & CAC & His & TAC & Tyr & 2 \\
\hline & & & & & GAC & Asp & 9,10 \\
\hline & & 425 & TCG & Ser & ATG & Met & 9,10 \\
\hline & & & & & TTG & Leu & 2,9 \\
\hline & & & & & TTC & Phe & $11,2,6,7,3$ \\
\hline & & 427 & CTG & Leu & CCG & Pro & 2 \\
\hline \multirow[t]{2}{*}{ Ofloxacin } & gyrA & 89 & GGC & Gly & TGC & Cys & 2 \\
\hline & & 91 & GCA & Ala & GTA & Val & $12,2,6$ \\
\hline
\end{tabular}

${ }^{\star}$ AA, amino acid.

$\dagger$ 1, Lee et al. (2001); 2, Maeda et al. (2001); 3, Williams et al. (1994); 4, Matsuoka et al. (2007); 5, Kai et al. (1999); 6, Matsuoka et al. (2000); 7 , Matsuoka et al. (2003); 8, Zhang et al. (2004); 9, Cambau et al. (2001); 10, Honoré \& Cole (1993); 11, Honoré et al. (1993); 12, Cambau et al. (1997). 
Table 2. Sequences of oligonucleotide primers for $M$. leprae

\begin{tabular}{|lllc|}
\hline Gene & \multicolumn{1}{c|}{ Primer } & \multicolumn{1}{c|}{ Sequence $\left(\mathbf{5}^{\prime} \mathbf{3}^{\prime}\right)$} & PCR products $(\mathbf{b p})$ \\
\hline \multirow{2}{*}{ folP1 } & MLfolP1DA-Ft; & GTGAGTTTGGCGCCAGTGCA & 119 \\
& MLfolP1DA-RB; & Biotin-GCAAGTTCTTTTACGACAGG & \\
rpoB & MLrpoBDA-F; & TCGCCGCTATCAAGGAATTC & 127 \\
& MLrpoBDA-RB; & Biotin-TCACGCGACAAACCACCCGG & \\
gyrA & MLgyrADA-F; & TGAGACTCCGGTTTCCGCCC & 139 \\
& MLgyrADA-RB; & Biotin-CAGCGACCACGGCTGCGCC & \\
& & & \\
\hline
\end{tabular}

cases, three patients had been treated with the WHO MDT regimen for 2 years and the other six patients had received dapsone monotherapy or combined treatment with clofazimine and rifampicin. All cases had a positive bacterial index (BI) and were therefore, by definition, multibacillary. Genomic DNA templates were prepared as described previously (Matsuoka et al. 2005, 2007). Briefly, slit-skin smear specimens were collected from the skin lesions of patients in the same manner as the routine procedure for BI determination. The bacilli were washed out from the blade into $70 \%$ ethanol and collected as a pellet by centrifugation at $10000 \mathrm{~g}$ for $20 \mathrm{~min}$. Genomic DNA templates for PCR were prepared by treatment with a lysis buffer as described elsewhere (de Wit et al., 1991). The LDS-DA assays were performed as described above and the results were translated into nucleotide sequences according to the positions of the spots for comparison with the sequence data.

Nucleotide sequencing. To confirm and verify the results obtained by the LDS-DA method, nucleotide sequences of PCR products were determined with the BigDye Terminator v1.1 Cycle Sequencing kit (Applied Biosystems) using the same primers for PCR amplification with an ABI310 genetic analyser.

Ethical approval and consent. The study was approved by the institutional ethics committee of the National Institute of Infectious Diseases, Japan, and two local institutional review boards. Bacterial samples were collected after informed consent was obtained.

\section{RESULTS}

\section{Development of a DNA microarray for drug susceptibility testing of $M$. leprae}

The target regions of the genes with expected length, $119 \mathrm{bp}$ for folP1, $127 \mathrm{bp}$ for $r p o B$ and $139 \mathrm{bp}$ for $g y r A$, were amplified simultaneously by mutiplex PCR as shown in Fig. 2. Several oligonucleotides corresponding to each of the wild-type and mutant sequences of folP $1, r p o B$ and $g y r A$

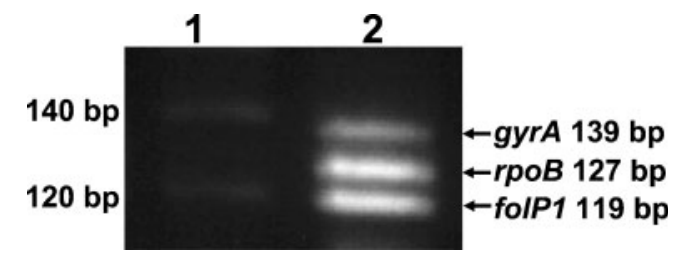

Fig. 2. Electrophoretic pattern obtained by multiplex $P C R$ for folP1, rpoB and gyrA. Lane 1, 20 bp ladder size markers; lane 2, PCR products. were synthesized, spotted on a glass slide and examined for hybridization with amplicons from the multiplex PCR. The best oligonucleotides, which hybridized with corresponding PCR products without reacting with others, were selected. A DNA microarray with selected oligonucleotides was established as presented in Fig. 1 and designated LDSDA. The performance of the LDS-DA was examined using PCR products from $M$. leprae isolates and artificially produced DNA fragments with known mutations. PCR products containing the drug-resistance-determining region (DRDR) for each gene were obtained by multiplex PCR (Fig. 2). Fig. 3 shows the hybridization patterns obtained from isolates grown in nude mice footpads, Thai-
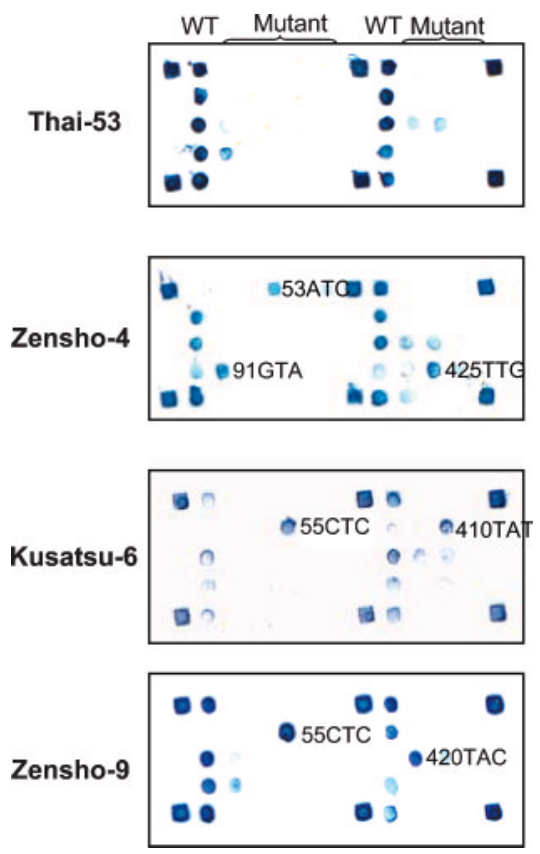

Fig. 3. Signals obtained by the LDS-DA with: a susceptible strain, Thai-53; strain Zensho-4, with mutation from ACC to ATC at codon 53 in the folP1 gene, from TCG to TTG at codon 425 in the rpo $B$ gene and from GCA to GTA at codon 91 in the gyrA gene; strain Kusatsu-6, with mutation from CCC to CTC at codon 55 in the folP1 gene and from GAT to TAT at codon 410 in the rpo $B$ gene; and strain Zensho-9, with mutation from CCC to CTC at codon 55 in the folP1 gene and from CAC to TAC at codon 420 in the $r p o B$ gene. 
53, Zensho-4 (Matsuoka et al., 2000) and two other strains with known nucleotide mutations (Zhang et al., 2004; Maeda et al., 2001). In Thai-53, which is susceptible to dapsone, rifampicin and ofloxacin, positive signals were observed on all of the wild-type spots. In contrast, the highest colour intensity was seen on the spot with the mutant oligonucleotide in drug-resistant $M$. leprae. In Zensho-4, with a three-drug-resistant phenotype, three positive signals shifted from the wild-type to the mutant spots. In the dapsone field, a mutation at codon 53 was identified by a positive signal on spot FM3 instead of FW1. In the ofloxacin and rifampicin fields, similar events were observed. Spots corresponding to mutant-type and wildtype sequences were also found in the other two isolates. Likewise, all the spots with mutant oligonucleotides were verified as to their proper reactivity with the PCR products carrying corresponding known mutations (data not shown).

\section{Evaluation of the LDS-DA system in two countries with high leprosy prevalence}

The LDS-DA system was successfully transferred to a laboratory in Yangon, Myanmar, and a laboratory in Cebu, the Philippines. The BI values of the samples from Myanmar varied from 1 to 6 . Most were more than 3 . Almost all samples from the Philippines showed a BI of more than 4, with a few samples of BI 2. Positive PCR results were obtained even from samples with a BI of 1 , although it was usually hard to obtain good results from PCR and colouring from samples with a BI of less than 3. One of the relapsed cases from Myanmar harboured $M$. leprae with mutations CCC to CGC at position 55 in the folP1 gene and TCG to ATG at position 425 in the rpoB gene. One resistant isolate with the mutation ACC to GCC at position 53 in the folP1 and another isolate with the mutation GAT to TAT at position 410 in the rpoB gene were new cases. In the samples from the Philippines, three $M$. leprae with mutations in the folP1 gene, CCC to CTC and CCC to TCC at position 55, were from relapsed cases. Two resistant isolates with mutation CCC to CGT at position 55 in the folP1 were from new cases. The results obtained by the LDS-DA system in these laboratories were compared with the nucleotide sequences of the corresponding genes, as shown in Table 3. All the samples possessing wild-type sequences were judged to be wild-type by both the LDS-DA and sequencing. Concordant results were also observed with seven specimens carrying mutations, five in Myanmar and two in the Philippines. Two unclear results were obtained in folP1 in Myanmar and one in $r p o B$ in the Philippines. In these three samples, the signals were not strong enough to be judged. In the row of codon 55 in folP1, no signal was observed with two specimens in the Philippines. Overall, the concordance between the LDS-DA and sequencing results on folP1 in Myanmar and the Philippines is $96.8 \%$ (61/63) and $97.3 \%$ (71/73), respectively. The LDS-DA results on $r p o B$ exhibited good agreement with sequencing results, $100 \%$ (63/63) and $98.6 \%(72 / 73)$ in Myanmar and the Philippines, respectively. No discordance was found between the LDS-DA and sequencing results on gyrA in either country (Table 4).

\section{DISCUSSION}

Detection of drug resistance in M. leprae is crucial for the efficient treatment of leprosy and the prevention of the spread of drug-resistant strains. The elucidation of the genetic background of resistance by molecular methods has enabled the prediction of drug susceptibility of $M$. leprae. Drug resistance to dapsone, rifampicin and ofloxacin has evolved by mutation in the DRDR in the folP $1, r p o B$ and gyrA genes respectively (Williams \& Gillis, 2004). A total of

Table 3. Comparison of results obtained by the LDS-DA and sequencing on clinical specimens in Myanmar and the Philippines

\begin{tabular}{|c|c|c|c|c|c|c|c|c|c|c|}
\hline \multirow[t]{2}{*}{ Study site } & \multirow[t]{2}{*}{ Status } & \multicolumn{3}{|c|}{ folP1 } & \multicolumn{3}{|c|}{$r p o B$} & \multicolumn{3}{|c|}{ gyrA } \\
\hline & & $\begin{array}{c}\text { No. of } \\
\text { specimens }\end{array}$ & LDS-DA & Sequence & $\begin{array}{c}\text { No. of } \\
\text { specimens }\end{array}$ & LDS-DA & Sequence & $\begin{array}{c}\text { No. of } \\
\text { specimens }\end{array}$ & LDS-DA & Sequence \\
\hline \multirow[t]{3}{*}{ Myanmar } & Concordant & 59 & $\mathrm{WT}^{\star}$ & WT & 61 & WT & WT & 62 & WT & WT \\
\hline & & 1 & $53: \mathrm{GCC} \dagger$ & 53 : GCC & 1 & $410:$ TAT & $410:$ TAT & 1 & $91:$ GTA & $91:$ GTA \\
\hline & & 1 & $55:$ CGC & $55:$ CGC & 1 & 425: ATG & 425 : ATG & & & \\
\hline \multirow{3}{*}{ Philippines } & & 2 & $55:$ CTC & 55 : CTC & & & & & & \\
\hline & & 1 & $55:$ TCC & $55:$ TCC & & & & & & \\
\hline & Discordant & 2 & 55 : nulls & 55 : CGT & 1 & Unclear & WT & & & \\
\hline
\end{tabular}

${ }^{\star}$ Wild-type sequence.

$\dagger$ Codon number: codon sequence.

$\$$ Data could not be translated because of weak signals.

$\S$ No signal was observed on the spots in raw folP1 55. 
Table 4. Concordance of LDS-DA results with sequencing in clinical specimens in Myanmar and the Philippines

\begin{tabular}{|lcrr|}
\hline \multirow{2}{*}{ Study site } & \multicolumn{3}{c|}{ Target gene } \\
\cline { 2 - 4 } & \multicolumn{1}{c|}{ folP1 } & \multicolumn{1}{c|}{$\boldsymbol{r p o B}$} & \multicolumn{1}{c|}{$\boldsymbol{g y r A}$} \\
\hline Myanmar & $61 / 63(96.8 \%)$ & $63 / 63(100 \%)$ & $63 / 63(100 \%)$ \\
The Philippines & $71 / 73(97.3 \%)$ & $72 / 73(98.6 \%)$ & $73 / 73(100 \%)$ \\
Total & $132 / 136(97.1 \%)$ & $135 / 136(99.3 \%)$ & $136 / 136(100 \%)$ \\
\hline
\end{tabular}

106 isolates without mutation in the $r p o B$ gene and 63 isolates without mutation the gyrA gene were susceptible to rifampicin and ofloxacin, respectively. All isolates resistant to rifampicin or ofloxacin harboured mutations in the DRDR of $r p o B$ or gyrA, respectively (Honoré \& Cole, 1993; Honoré et al., 1993; Williams et al., 1994, 2001; Cambau et al., 1997, 2001; Matsuoka et al., 2000, 2003; Maeda et al., 2001; Zhang et al., 2004). Resistance of $M$. leprae to dapsone in the mouse footpad is classified into three degrees, namely, low, intermediate and high. A total of 84 isolates without mutation in the folP 1 gene were susceptible to dapsone, but one isolate was resistant with intermediate degree and five isolates were resistant with low degree (Cambau et al., 2006). On the other hand, a total of 24 isolates resistant to dapsone with high or intermediate degree revealed amino acid substitution at the DRDR of the fop1 gene (Kai et al., 1999; Matsuoka et al., 2000, 2003, 2007; Williams et al., 2000; Lee et al., 2001; Maeda et al., 2001; Cambau et al., 2006). An isolate with mutation ACC to GCC at codon 53 was demonstrated to be resistant with low degree (Cambau et al., 2006), though it is not clear whether dapsone resistance with low degree is true resistance (Matsuoka et al., 2007). Other isolates with this mutation were found to be resistant to dapsone with intermediate degree. Therefore contradiction between mutation in the folP1 gene and the results obtained by the mouse footpad drug susceptibility test has been encountered for only one case so far.

Although the direct sequencing of PCR products is definitive and allows rapid detection of resistant cases, it has the disadvantage of requiring expensive apparatus and high sequencing costs, so it is not practical in many developing countries. The heteroduplex method (HAD) (Williams et al., 2001) and the PCR-single-strand conformation polymorphism method (SSCP) (Honoré et al., 1993) have been applied to the detection of mutants to overcome these disadvantages. The HAD method can identify mutations in the PCR-amplified fragments by the electrophoretic mobility difference of heteroduplexes of wild-type products and test sample products, while the SSCP method analyses that of single-stranded products. However, neither the HAD nor the SSCP method fully meets the required conditions in developing countries, since these methods demand complicated procedures and both detect silent mutations as resistant mutations. The recently developed LineProbe assay based on reverse hybridization can detect rifampicin-resistant $M$. leprae simply and rapidly, but it cannot provide susceptibility information for other anti-leprosy drugs. The multipleprimer PCR amplification refractory mutation system is relatively simple but detects only nucleotide mutations and cannot distinguish silent mutations from missense mutations (Sapkota et al., 2008)

Our present study aimed to exploit a rapid, simple and simultaneous drug susceptibility test for three key antileprosy drugs to solve defects of each method previously reported, based on DNA-DNA hybridization using a DNA microarray. The novel method, designated LDS-DA, allows the simultaneous identification of mutations in three genes, responsible for resistance to dapsone, rifampicin and the quinolones. Easy accessibility and high reproducibility demonstrated by the studies with clinical materials in two developing countries revealed the superior applicability of this method. Only five discordant results were found in 136 specimens examined. Three discordant results, two in Myanmar on folP1 and one in the Philippines on $r p o B$, showed faint reactions on multiple spots probably caused by some technical errors. In the remaining two discordant results found in the Philippines, no signal was found at any position in row 55 of folP1. These samples were shown to carry a mutation at codon 55 in folP 1 from the wild-type CCC to CGT (Table 2), which was recently revealed to be associated with dapsone resistance (Cambau et al., 2006) and was not covered by the oligonucleotide array on the LDS-DA. Although the signal was found neither at the wild-type nor at the mutant position in row 55 of $f_{0} l P 1$, this result can be taken as suggestive of dapsone resistance. The absence of a positive signal in the wild-type position implies the existence of base substitutions in the region covered by the oligonucleotide. Similar translation criteria have been applied to rifampicin-resistant $M$. tuberculosis by the commercially available INNO-LiPA Rif TB assay (Rossau et al., 1997). Other possible mutation(s) related to drug resistance can also be distinguished under the same criteria.

The monitoring of drug-resistant leprosy cases has been recommended in order to maintain the effectiveness of chemotherapy for leprosy (Ji, 2002; Matsuoka et al., 2007). The LDS-DA method developed in this study seems to be a simple and robust tool to assess the drug susceptibility of M. leprae in developing countries, where susceptibility testing is rarely applied. Comprehensive data on the prevalence of resistant cases shows that the level of drug resistance is low in some endemic countries (Matsuoka et al., 2007). It is therefore recommended to apply this method to samples from intractable cases and relapsed cases, to examine the susceptibility to anti-leprosy drugs and ensure effective treatment. Additionally, the capacity of the LDS-DA method to identify the positions of mutations can be utilized for molecular epidemiological and geographical studies on the spread of drug-resistant M. leprae. 


\section{ACKNOWLEDGEMENTS}

This study was supported by the following grants: a Health Research Grant for Emerging and Re-emerging Infectious Diseases, Ministry of Health, Labour and Welfare, Government of Japan; a grant from the International Medical Center, Ministry of Health, Labour and Welfare; a grant from the US-Japan Cooperative Medical Science Programs; and also by the Grants-in-Aid Program of the Founding Research Center for Emerging and Reemerging Infectious Diseases from the Ministry of Education, Culture, Sports, Science, and Technology, Japan, to Y.S.

\section{REFERENCES}

Cambau, E., Perani, E., Guillemin, I., Jamet, P. \& Ji, B. (1997). Multidrug rtesistance to dapsone, rifampicin, and ofloxacin in Mycobacterium leprae. Lancet 349, 103-104.

Cambau, E., Bonnafous, P., Perani, E., Sougakoff, W., Ji, B. \& Jarlier, V. (2001). Molecular detection of rifampicin and ofloxacin resistance for patients who experience relapse of multibacillary leprosy. Clin Infect Dis 34, 39-45.

Cambau, E., Carthagena, L., Chauffour, A., Ji, B. \& Jarlier, V. (2006). Dihydropteroate synthase mutations in the folP1 gene predict dapsone resistance in relapsed cases of leprosy. Clin Infect Dis 42, 238-241.

de Wit, M. Y. L., Faber, W. R. S., Krieg, R., Douglas, J. T., Lucas, S. B., Montreewasuwat, N., Pattyn, S. R. N., Hussain, R., Ponnighaus, J. M. $\&$ other authors (1991). Application of polymerase chain reaction for the detection of Mycobacterium leprae in skin tissue. J Clin Microbiol 29, 906-910.

Honoré, N. \& Cole, S. T. (1993). Molecular basis of rifampicin resistance in Mycobacterium leprae. Antimicrob Agents Chemother 37, 414-418.

Honoré, N., Perani, E., Telenti, A., Grosset, J. \& Cole, S. T. (1993). A simple and rapid technique for the detection of rifampicin resistance in Mycobacterium leprae. Int J Lepr Other Mycobact Dis 61, 600-604.

Ji, B. (2002). Rifampicin resistant leprosy: a review and research proposal of a pilot study. Lepr Rev 73, 2-8.

Kai, M., Matsuoka, M., Nakata, N., Maeda, S., Gidoh, M., Maeda, Y., Hashimoto, K., Kobayashi, K. \& Kashiwabara, Y. (1999). Diaminodiphenilsulfone resistance of Mycobacterium leprae due to mutations in the dihydropteroate synthase gene. FEMS Microbiol Lett 177, 231-235.

Lee, S. B., Kim, S. K., Kang, T. J., Chae, G. T., Chun, J. H., Shin, H. K., Kim, J. P., Ko, Y. H. \& Kim, N. H. (2001). The prevalence of folP1 mutations associated with clinical resistance to dapsone, in Mycoabcterium leprae isolates from South Korea. Ann Trop Med Parasitol 95, 429-432.

Maeda, S., Matsuoka, M., Nakata, N., Kai, M., Maeda, Y., Hashimoto, K., Kimura, H., Kobayashi, K. \& Kashiwabara, Y. (2001). Multidrug resistant Mycobacterium leprae from patients with leprosy. Antimicrob Agents Chemother 45, 3635-3639.

Matsuoka, M., Kashiwabara, Y. \& Namisato, Y. (2000). A Mycobacterium leprae isolate resistant to dapsone, rifampicin, ofloxacine and sparfloxacin. Int J Lepr Other Mycobact Dis 68, $452-455$.

Matsuoka, M., Kashiwabara, Y., Zhang, L., Gotoh, M. \& Kitajima, S. (2003). A second case of multidrug resistant Mycobacterium leprae isolated from a Japanese patient with relapsed lepromatous leprosy. Int J Lepr Other Mycobact Dis 71, 240-243.

Matsuoka, M., Zhang, L., Morris, M. F., Legua, P. \& Wiens, C. (2005). Polymorphism in the rpoT gene in Mycobacterium leprae isolates obtained from Latin American countries and its possible correlation with the spread of leprosy. FEMS Microbiol Lett 243, 311-315.

Matsuoka, M., Teky, B., Khin, S. A., Kyaw, K., Tan, E. V., dela Cruz, E., Gelber, R., Saunderson, P., Balagon, M. A. \& Pannikar, V. (2007). The frequency of drug resistance mutations in Mycobacterium leprae isolates in untreated and relapsed leprosy patients from Myanmar, Indonesia and the Philippines. Lepr Rev 78, 343-352.

Rossau, R., Traore, H., de Beenhouwer, H., Mijs, W., Jannes, G., De Rijk, P. \& Portaels, F. (1997). Evaluation of the INNO-LiPA Rif. TB assay, a reverse hybridization assay for the simultaneous detection of Mycobacterium tuberculosis complex and its resistance to rifampicin. Antimicrob Agents Chemother 41, 2093-2098.

Sapkota, B. R., Ranjit, C., Neupane, K. D. \& Macdonald, M. (2008). Developing and evaluation of a novel multiple-primer PCR amplification refractory mutation system for the rapid detection of mutations conferring rifampicin resistance in codon 425 of the rpoB gene of Mycobacterium leprae. J Med Microbiol 57, 179-184.

Shepard, C. (1960). The experimental disease that follows the injection of human leprosy bacillus into footpads of mice. J Exp Med 112, 445-454.

WHO (1998). WHO Expert Committee on Leprosy, seventh report. Technical report series 87. Geneva: World Health Organization.

Williams, D. L. \& Gillis, T. P. (2004). Molecular detection of resistance in Mycobacterium leprae. Lepr Rev 75, 118-130.

Williams, D. L., Waguespack, C., Eisenack, K., Crawford, J. T., Portaels, F., Salfinger, M., Nolan, M. C., Abe, C., Stich-Groh, V. \& Gillis, T. P. (1994). Characterization of rifampicin resistance in pathogenic mycobacteria. Antimicrob Agents Chemother 38, 23802386.

Williams, D. L., Spring, L., Harris, E., Roche, P. \& Gillis, T. P. (2000). Dihydropteroate synthase of Mycobacterium leprae and dapsone resistance. Antimicrob Agents Chemother 44, 1530-1537.

Williams, D. L., Pittman, T. L., Gillis, T. P., Matsuoka, M. \& Kashiwabara, Y. (2001). Simultaneous detection of Mycobacterium leprae and its susceptibility to dapsone using DNA heteroduplex analysis. J Clin Microbiol 39, 2083-2088.

Zhang, L., Namisato, M. \& Matsuoka, M. (2004). A mutation at codon 516 in the rpoB gene of Mycobacterium leprae confers resistance to rifampicin. Int J Lepr Other Mycobact Dis 72, 468-472. 